\title{
NEMO-Enabled Localized Mobility Support for Internet Access in Automotive Scenarios
}

\author{
Ignacio Soto, Carlos J. Bernardos, Maria Calderon, and Albert Banchs, University Carlos III of Madrid \\ Arturo Azcorra, University Carlos III of Madrid and IMDEA Networks
}

\footnotetext{
${ }^{1}$ Some examples are the work in the IETF MEXT WG (http:// www.ietf.org/html.charters/mext-charter.html), the extension by the ETSI Technical Committee Railways Telecommunications

(http://portal.etsi.org/rt/su mmary_06.asp) of the original global system for mobile communicationsrailway $(G S M-R)$ standard to benefit from the evolution of the GSM technology, or the Partners for Advanced Transit and Highways (PATH) initiative

(http://www.path.berkeley.edu/PATH/

Research/currenttransit.ht $\mathrm{ml}$ ), which among other goals conducts research in technologies for innovating and enhancing public transportation solutions.
}

\begin{abstract}
This article surveys the major existing approaches and proposes a novel architecture to support mobile networks in network-based, localized mobility domains. Our architecture enables conventional terminals without mobility support to obtain connectivity either from fixed locations or mobile platforms (e.g., vehicles) and move between them, while keeping their ongoing sessions. This functionality offers broadband Internet access in automotive scenarios such as public transportation systems, where users spend time both in vehicles and at stations. The key advantage of our proposal, as compared with current alternatives, is that the described mobile functionality is provided to conventional IP devices that lack mobility functionality. We also performed an experimental evaluation of our proposal that shows that our architecture improves the quality perceived by the end users.
\end{abstract}

\section{INTRODUCTION}

Nowadays, users increasingly demand Internet access everywhere. The current trend in handheld terminals is toward devices that move away from the traditional phone service model and incorporate a large number of different data applications. Equipping terminals with multiple technologies - for example, third generation $(3 \mathrm{G})$ and wireless local area network (WLAN) - is a widely used solution to provide ubiquitous Internet access. Internet access in automotive scenarios is a particularly relevant case, especially because people in modern cities spend a lot of time in vehicles. Although $3 \mathrm{G}$ is a possible option, it suffers from a number of drawbacks, such as capacity constraints from the point of view of the operator, as well as cost issues from the end-user perspective.

In the above context, there is a need for an alternative solution to $3 \mathrm{G}$ that provides efficient broadband Internet access in automotive scenarios. Public transportation systems, such as under- grounds, suburban trains, and city buses, represent one relevant scenario because of the large number of users and the time spent by these users both in vehicles and stations. In fact, communications in these environments are receiving a lot of attention from a number of research and standardization activities. ${ }^{1}$ Other relevant scenarios with similar requirements are those in which users move around large areas (e.g., airports, exhibition sites, or fairgrounds). In these areas, attachment points to the Internet might be available both in fixed locations (such as coffee shops or airport terminals) or in mobile platforms, such as vehicles (e.g., buses that move between pavilions at a fair or a train that moves from one terminal to another at an airport). Users demand the ability to keep their ongoing communications while changing their point of attachment to the network as they move around (e.g., when a user leaves a coffee shop and gets on a bus).

Currently, NEtwork MObility (NEMO) solutions are being developed by the Internet Engineering Task Force (IETF) and the research community to offer Internet access from vehicles. Special devices (called mobile routers [MRs]), located in the vehicles, handle the communication with the fixed infrastructure and provide access to passengers' devices using a convenient short-range radio technology. However, in the scenarios mentioned above, users spend only part of their time in the vehicles because they also move from vehicles to fixed platforms (e.g., the stations in the public transportation scenario or the terminals in the airport scenario). Therefore, an integrated solution for these scenarios, which considers Internet access not only from vehicles but also from associated fixed platforms, is a better approach.

Traditional Internet Protocol (IP) mobility mechanisms $[1,2]$ were based on functionality residing both in the moving terminals and in the network. Lately, there is a new trend toward solutions that enable the mobility of IP devices within a local domain with only support from the network. This approach, called network-based 
localized mobility management (NetLMM) [3], allows conventional IP devices to benefit from this mobility support. This is very interesting from the point of view of operators because it allows them to provide mobility support without depending on software and complex mobilityrelated configuration in the terminals. The IETF has standardized Proxy Mobile IPv6 (PMIPv6) [4], a protocol to provide this functionality. But this solution has the limitation of not fully supporting mobile networks.

In this article we propose a novel architecture, called NEMO-enabled PMIPv6 (NPMIPv6), which fully integrates mobile networks in PMIPv6-localized-mobility domains. With our approach, users can obtain connectivity either from fixed locations or mobile platforms (e.g., vehicles) and can move between them while keeping their ongoing sessions. N-PMIPv6 architecture exhibits two remarkable characteristics. First, N-PMIPv6 is totally network-based therefore no mobility support is required in the terminals - and second, the handover performance is improved, both in terms of latency and signaling overhead.

\section{OVERVIEW OF}

\section{MAJOR EXISTING APPROACHES}

This section provides an overview of existing mechanisms developed by the IETF that are relevant for providing Internet access in vehicular environments. Operators have shown great interest in network-based localized mobility solutions. Additionally, NEMO approaches are a key element to provide connectivity from vehicles. Combining both brings the advantages of network-based, localized-mobility solutions to vehicular scenarios. This section reviews the work of the IETF in this area and highlights the limitations of current solutions.

\section{Network-BASed Localized MobiLity}

Unlike host-based localized mobility [1], where mobile terminals (MTs) signal a location change to the network to update routing states, NetLMM [3] approaches provide mobility support to moving hosts without their involvement. This is achieved by relocating relevant functionality for mobility management from the MT to the network. In a localized mobility domain (LMD), the network learns through standard terminal operation, such as router and neighbor discovery or by means of linklayer support, about the movement of an MT and coordinates routing state updates without any mobility-specific support from the terminal. While moving inside the LMD, the MT keeps its IP address, and the network is in charge of updating its location in an efficient manner. PMIPv6 [4] is the NetLMM protocol proposed by the IETF. This protocol is based on mobile IPv6 (MIPv6) [2] - it extends MIPv6 signaling messages and reuses the home agent (HA) concept.

The core functional entities in the PMIPv6 infrastructure are (Fig. 1):

- Mobile Access Gateway (MAG): This entity performs the mobility-related signaling on

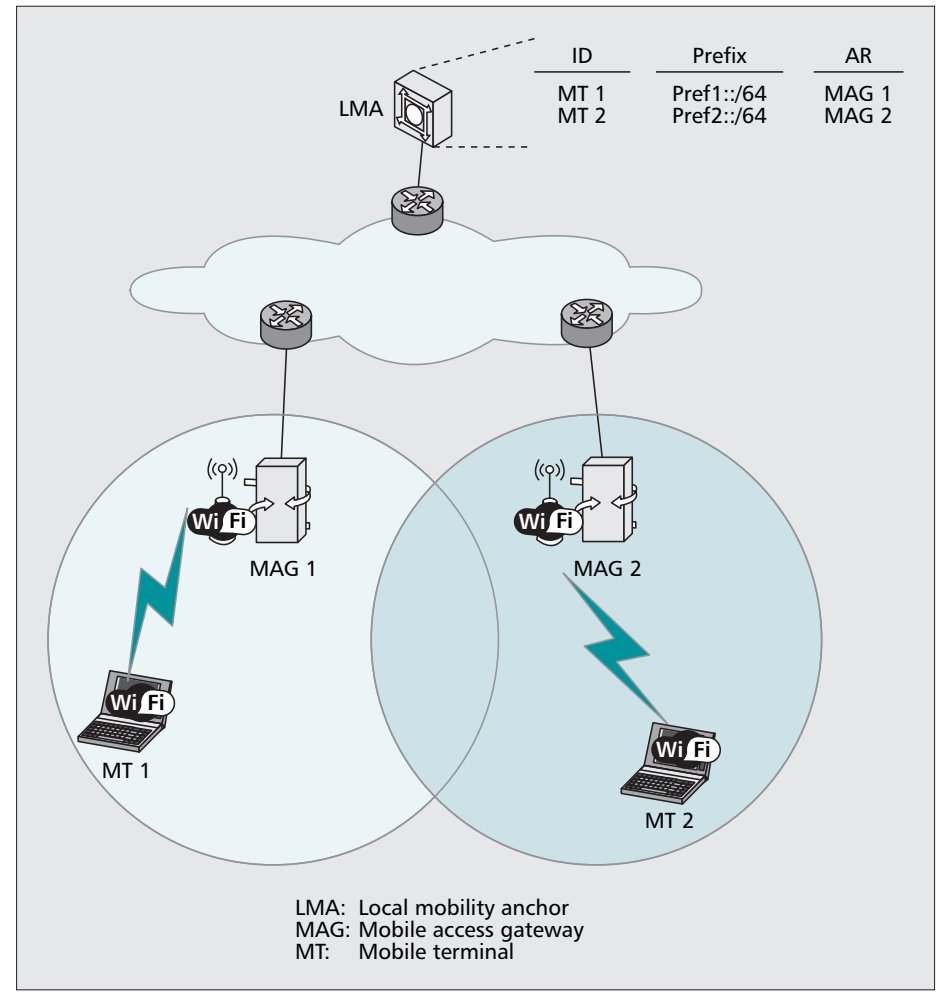

Figure 1. Proxy Mobile IPv6 domain.

behalf of an MT that is attached to its access link. The MAG is usually the access router for the MT, that is, the first hop router in the localized mobility management infrastructure. It is responsible for tracking the movements of the MT in the access link. There are multiple MAGs in an LMD.

- Local Mobility Anchor (LMA): This is an entity within the backbone network that maintains a collection of routes for individual MTs within the LMD. The routes point to the MAGs managing the links in which the MTs are currently located. Packets for an MT are routed to and from the MT through tunnels between the LMA and the corresponding MAG.

After an MT enters an LMD and attaches to an access link, the MAG in that access link, after identifying the MT, performs mobility signaling on behalf of the MT. The MAG sends a proxy binding update (PBU) to the LMA, associating its own address with the MT identity (e.g., its medium access control [MAC] address or an ID related with its authentication in the network). Upon receiving this request, the LMA assigns a prefix to the MT. Then, the LMA sends a proxy binding acknowledgment (PBA) including the prefix assigned to the MT to the MAG. It also creates a binding cache entry and establishes a bidirectional tunnel to the MAG. Whenever the MT moves, the new MAG updates the MT location in the LMA and advertises the same prefix to the MT (through unicast router advertisement messages), thereby making the IP mobility trans- 
parent to the MT. The MT can keep the address configured when it first entered the LMD, even after changing its point of attachment within the network.

\section{NETWORK MOBILITY SUPPORT}

To address the requirement of transparent Internet access from vehicles, the IETF standardized the NEMO Basic Support (NEMO B.S.) protocol [5]. This protocol defines a mobile network (or NEtwork that MOves [NEMO]) as a network whose attachment point to the Internet varies with time. The router within the NEMO that connects to the Internet is called the MR. It is assumed that the NEMO has a home network where it resides when it is not moving. Because the NEMO is part of the home network, the mobile network has configured addresses belonging to one or more address blocks assigned to the home network: the mobile network prefixes (MNPs). These addresses remain assigned to the NEMO when it is away from home, although they only have topological meaning when the NEMO is at home. So, when the NEMO is away from home, packets addressed to the mobile network nodes (MNNs) still will be routed to the home network. Additionally, when the NEMO is away from home, that is, it is in a visited network, the MR acquires an address from the visited network, called the care-of address (CoA), where the routing infrastructure can deliver packets without additional mechanisms.

The basic solution for network mobility support is quite similar to the solution proposed for host mobility (mobile IPv6 [2]) and essentially creates a bidirectional tunnel between a special node located in the home network of the NEMO (the HA) and the CoA of the MR. Currently, route optimization support is being researched, with special attention being paid to the requirements of the vehicular scenario [6].

\section{THE CURRENT SOlution FOR COMBINING NEMO AND PMIPv6}

Both the NEMO and NetLMM solutions provide interesting features that can be combined in an integrated architecture. Nowadays, it is possible to partially benefit from the following advantages by using NEMO B.S. and PMIPv6:

- Transparent network mobility support: MRs manage the mobility of a network composed of a set of devices moving together.

- Transparent localized mobility support without node involvement: MRs and MTs can roam within a PMIPv6 domain without changing their IP addresses.

Although current mechanisms (i.e., NEMO B.S. and PMIPv6) can be combined to provide the advantages described above, this combination does not constitute a full integration because an MT cannot roam between an MR and a MAG of the fixed infrastructure without changing its IP address. This is because the addresses used within the mobile network belong to the MNP and not to the prefixes used by PMIPv6. This means that to support - in a transparent way - MTs roaming between MRs and MAGs without any restriction, MTs are required to run MIPv6 to manage mobility (that is, the change of IP address) by themselves. If MTs must use MIPv6, the mobility support provided within the PMIPv6 domain is no longer fully network-based because some mobility operations are performed by MTs.

\section{N-PMIPV6 ARCHITECTURE}

In this section we propose a novel architecture that overcomes the shortcomings identified in the previous section for the current solution for NEMO support in PMIPv6. Our architecture, called N-PMIPv6, enables a seamless and efficient integration of mobile networks within a NetLMM solution, based on PMIPv6, without adding extra mobility support on terminals (i.e., mobility is totally managed by the network) and improving handover performance. First, an overview of the architecture is provided, and subsequently its operation is presented in greater detail.

\section{OVERVIEW}

The key idea of N-PMIPv6 consists in extending the PMIPv6 domain to also include mobile networks. Both the fixed infrastructure (i.e., MAGs) and the mobile networks (i.e., MRs) belong to the same network operator. With NPMIPv6, an MT attached to a mobile network is also part of the PMIPv6 domain. Hereinafter, we refer to an N-PMIPv6-enabled LMD as an N-PMIPv6 domain. This enables conventional IP nodes to roam between fixed MAGs and also between fixed MAGs and MRs, without changing the IPv6 addresses they are using. As a result, the handover-related signaling load is reduced, and the handover performance (i.e., the associated latency) is improved when compared to traditional global IP-mobility solutions (e.g., MIPv6).

Whereas the NEMO B.S. protocol requires MRs to manage their own mobility, this is not required in N-PMIPv6, in the same way that $\mathrm{N}$ PMIPv6 does not require mobility-related functionality in MTs. This is because the mobility of MRs and MTs in N-PMIPv6 is managed by the network (i.e., it is network-based). With NPMIPv6, MTs do not require additional functionality. MRs require functionality to extend the PMIPv6 domain to mobile networks so that an MT that attaches to a mobile network is not required to change its IPv6 address. Because MRs in N-PMIPv6 perform similar functions to MAGs in PMIPv6 while being mobile, hereafter we refer to them with the name moving MAGs (mMAGs).

The mMAGs extend the PMIPv6 domain by providing IPv6 prefixes belonging to this domain to attached MTs and by forwarding their packets through the LMA. The basic operation of an mMAG is as follows. When an mAG attaches to a fixed MAG, the fixed MAG informs its LMA about this event by sending a PBU message that contains the identity of the mMAG. The LMA then delegates an IPv6 prefix to the mMAG and creates a binding cache entry, associating the mMAG identity with the delegated prefix and the fixed MAG to which the mMAG is attached. If the mMAG moves to another 




Figure 2. Architecture overview of an N-PMIPv6 domain.

fixed MAG, the LMA updates the binding with the information of the new MAG. Note that this is basically the PMIPv6 behavior when a conventional MT connects to a PMIPv6 MAG, that is, our architecture manages the mobility of an mMAG in the same way that PMIPv6 manages the mobility of an MT.

From the point of view of an MT that attaches to an mMAG, this mMAG behaves as a fixed MAG of the N-PMIPv6 domain. In particular, when an MT attaches to an mMAG, the mMAG informs the LMA and, following PMIPv6 procedures, obtains an IPv6 prefix for the MT. The LMA then adds a new binding cache entry, associating the ID of the MT with the delegated prefix and the MAG IPv6 address to which it is attached (i.e., the mMAG address). The LMA cannot accept requests for these kinds of operations from any node, only from authorized MAGs. This implies that mMAGs must have a security association with the LMAs to be able to operate in the N-PMIPv6 domain. The way this association is created is beyond the scope of this article, but note that it is not different from the security association required with any fixed MAG. This basically means that for practical purposes, we assume scenarios in which the mMAGs, the fixed MAGs, and the LMA belong to the same administrative domain, as would be the case in the automotive scenarios described in the introduction.

To deliver IPv6 packets addressed to an MT attached to a connected mMAG, a change in the normal operation of a PMIPv6 LMA is introduced. Specifically, we extend LMA functionality to support recursive look ups in its binding cache as follows. In a first look up, the LMA obtains the mMAG to which the MT is attached. After that, the LMA performs a second look up searching for this mMAG in its binding cache, and finds the associated fixed MAG. With this information, the LMA can encapsulate the received packets toward the $\mathrm{mMAG}$, through the appropriate fixed MAG. Then, the mMAG can forward data packets to the MT. Two nested tunnels are used to encapsulate data packets between the LMA and the mMAG: one between the LMA and the mMAG and another one between the LMA and the fixed MAG. A new field, called mMAG (M) flag, is added to the binding cache used by the LMA to support recursive look ups. The entries in the binding cache created/updated by PBUs received from mMAGs have the M flag set to "yes." On the other hand, entries created/updated by PBUs received from fixed MAGs have the $M$ flag set to "no." The use of this flag avoids having the LMA perform unnecessary recursive look ups in its binding cache.

\section{Detalled Operation}

This section describes in more detail the operation of the N-PMIPv6 architecture, using the network scenario that appears in Fig. 2 and the signaling sequence depicted in Fig. 3. 


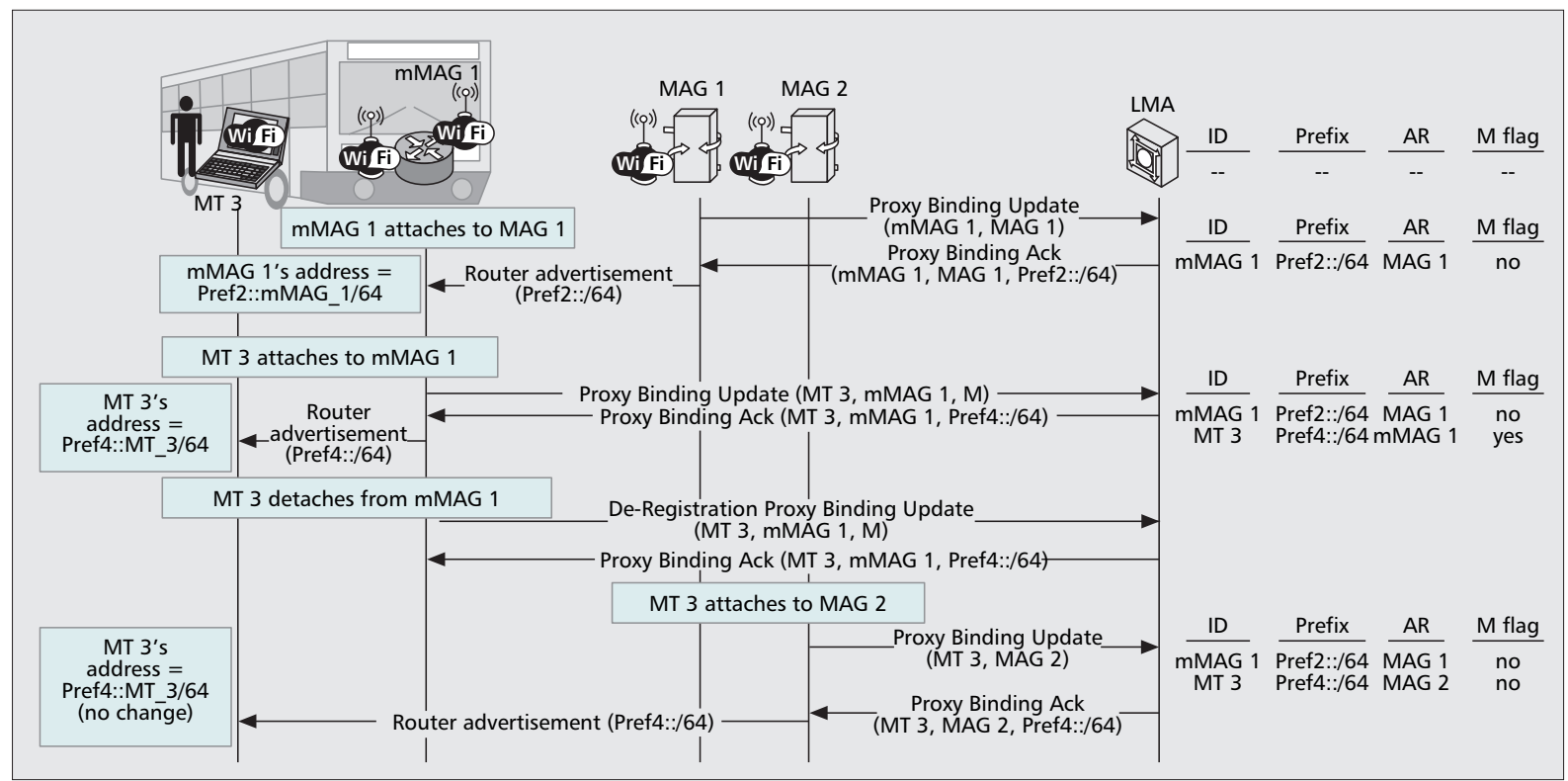

Figure 3. Detailed operation signaling.

When an mMAG - mMAG 1 - attaches to a fixed MAG - MAG 1 - this event is detected by MAG 1 and reported to its serving LMA by means of a PBU message. If no existing entry for mMAG 1 is found in the LMA binding cache, the LMA assigns an IPv6 prefix to the mMAG 1 (Pref $2:: / 64$ ) and creates a new entry in the cache. This entry includes the information of the assigned IPv6 prefix and the IPv6 address of the fixed MAG to which mMAG 1 is attached (i.e., MAG 1). The LMA then replies with a PBA message that includes the IPv6 prefix assigned to mMAG 1 (Pref $2:$ : /64). With this information, MAG 1 sends a unicast router advertisement (RA) message to mMAG 1 so it can form an IPv6 address and start sending/receiving traffic. While the mMAG moves within the same domain - roaming between different fixed MAGs - its IPv6 address does not change.

When an MT - MT 3 - attaches to mMAG 1, mMAG 1 sends a PBU message toward the LMA, which assigns an IPv6 prefix to MT 3 (Pref $4::$ /64) and creates a new entry for this MT in its binding cache, setting the $M$ flag of this entry to "yes." 2 The LMA then provides mMAG 1 with the assigned prefix. Finally, mMAG 1 informs MT 3 about the IPv6 prefix it must use by sending a unicast RA to the MT.

To hide the network topology and avoid changing the particular prefix assigned to an mMAG or an MT while they roam within the same domain, IP bidirectional tunneling is used. Following our example, if the LMA receives a packet from a correspondent node (CN) addressed to MT 3, it performs a recursive look up at its binding cache. As a result of this look up, the packet is sent through a nested tunnel, the inner header with the source address set to the LMA and destination address, the mMAG 1 , and the outer header with source address the LMA and destination address, the MAG 1 . The outer header brings the packet to MAG 1, which then removes that header. Next, the inner header brings the packet to the mMAG 1 . Finally, mMAG 1 removes the inner header and delivers the packet to MT 3.

If MT 3 performs an intra N-PMIPv6 domain handover from mMAG 1 to MAG 2 (Fig. 3), MAG 2 informs the LMA so it can update the binding entry accordingly (now MT 3 is attached to MAG 2 instead of mMAG 1, and the M flag is set to "no"). The mMAG 1, upon detecting disconnection of MT 3, sends a deregistration PBU (a PBU with the lifetime value of zero) to its LMA, following standard PMIPv6 operation. If the LMA does not receive a PBU about MT 3 after a pre-configured amount of time, the binding entry is deleted to avoid a stale state at the LMA binding cache.

\section{SCALABILITY OF THE SOLUTION}

An additional advantage of our proposal as compared with PMIPv6 is that it increases the scalability because mMAGs concentrate MTs. Therefore, when a vehicle moves, instead of a number of individual MTs changing their point of attachment to the network with a control message per MT sent by the MAG to the LMA, we have just one control message sent by the MAG to the LMA, indicating the movement of the mMAG. The cost, from the point of view of scalability, is having more entries (one per mMAG) in the binding cache of the LMA, but this is not a problem as it is always possible to distribute the LMA function among different nodes in the network.

\section{Performance Evaluation}

In this section we evaluate the performance improvement achieved with N-PMIPv6 when compared with the existing approach for NEMO support in PMIPv6 domains (NEMO+MIPv6+PMIPv6) described previously 


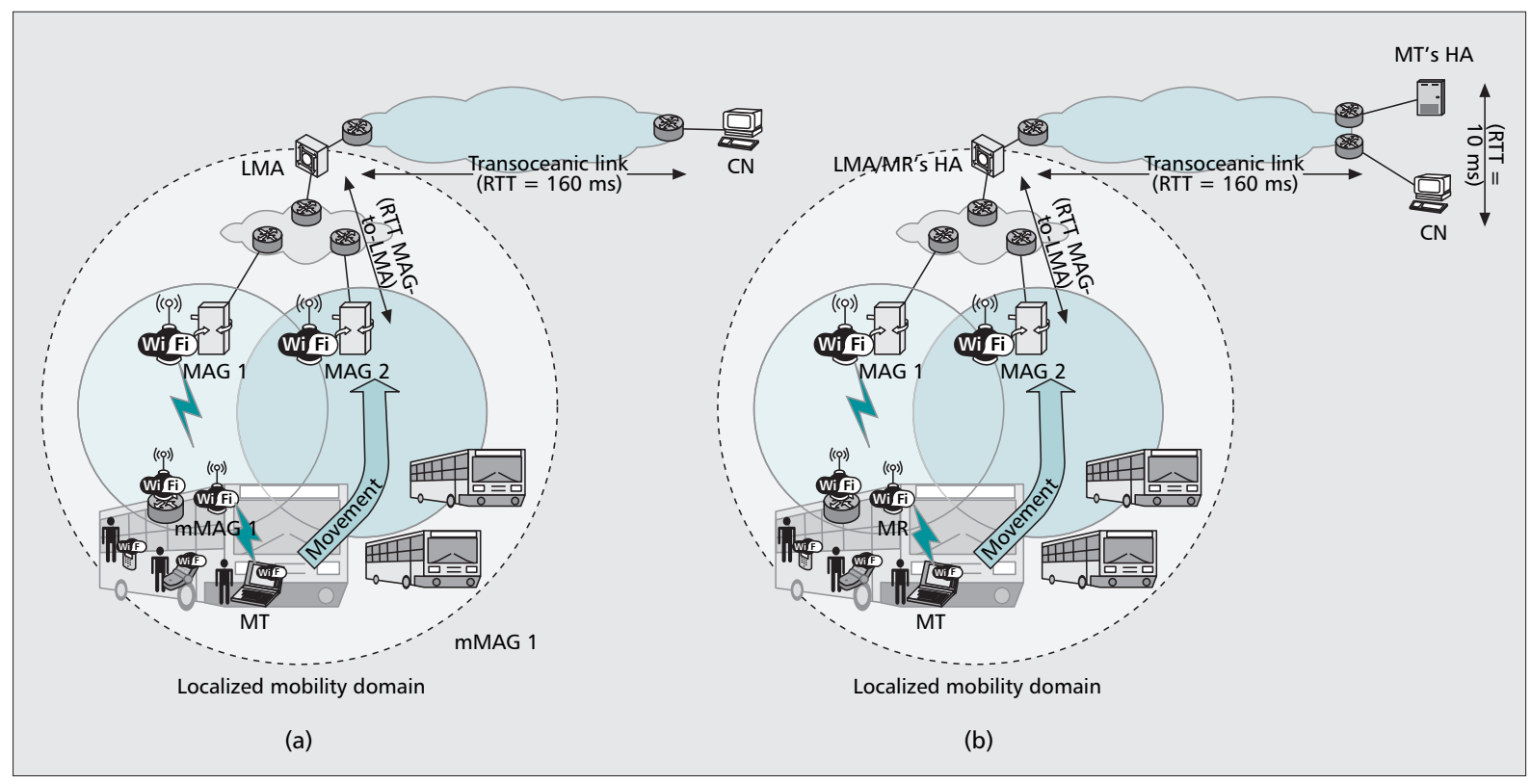

Figure 4. Analyzed scenarios: a) N-PMIPv6; b) NEMO+MIPv6+PMIPv6.

in the overview of existing approaches. The main benefit of N-PMIPv6 over NEMO + MIPv6 + PMIPv6 is that N-PMIPv6 does not require mobility support on terminals. Moreover, in this section we show that this benefit comes at no performance penalty and that $\mathrm{N}$ PMIPv6 actually provides better performance than NEMO+MIPv6+PMIPv6.

Figure 4 shows the two scenarios we consider in this section. The left part shows an N-PMIPv6 domain consisting of two MAGs, one LMA, one mMAG, and one MT. The right part shows a network deployment of the NEMO+MIPv6+PMIPv6 approach, consisting of two MAGs, one LMA, one MR and its HA (called MR's HA), and one MT and its HA (MT's HA). In both scenarios, there is a CN located on the Internet communicating with the MT.

From the point of view of performance, the key advantage of N-PMIPv6 over NEMO+MIPv6+PMIPv6 is that upon executing an MT handover to or from a mobile network, the corresponding signaling is sent only to the LMA, as opposed to NEMO+MIPv6+PMIPv6, which requires signaling down to the MT's HA. This results in a reduction of the signaling load in the backbone, as well as shorter handover latencies.

In the case of an mMAG/MR handover, because mobility is managed by PMIPv6 (i.e., the location of the mMAG/MR is updated at the LMA by the MAG to which the mMAG/MR is attached, and no further signaling is required) in both N-PMIPv6 and NEMO+MIPv6+PMIPv6 solutions, the handover performance is the same.

In this section we concentrate on the performance analysis for the case of the MT handover because this is the only case in which the performance of both approaches differs.

In the NEMO+MIPv6+PMIPv6 scenario, the MT and its HA are separated by a transoceanic link in order to understand the impact of long round-trip times (RTTs) on performance. The MT is communicating with a $\mathrm{CN}$ that is topologically close to the MT's HA. The N-PMIPv6 scenario is equivalent in terms of functionality and the location of the relevant network entities. The LMA of the N-PMIPv6 scenario is located in the same place that the MR's HA in the NEMO+MIPv6+PMIPv6 scenario is in order to perform a fair comparison. The location of the MR's HA has an impact on the end-to-end delay of data traffic because every packet sent by a node attached to the MR must traverse the MR's HA (i.e., there is no standardized NEMO route optimization solution yet).

We estimate the MT-handover latency for both N-PMIPv6 - handovers from an mMAG to a MAG or vice versa - and NEMO + MIPv6 + PMIPv6 - handovers from MAG to MAG. We assume that in the NEMO+MIPv6+PMIPv6 case, the MT is performing MIPv6 route optimization (RO) with the CN so data packets do not traverse the MT's HA. The MT-handover latency can be estimated for this case following [7], according to which latency is approximately equal to one MT-HA RTT plus one MT-CN RTT, which is roughly two MT-HA RTTs (we take the RTT measurements of [8]), because of the return routability signaling required to perform $\mathrm{RO}$ with the $\mathrm{CN}$. For the N-PMIPv6 case, the handover latency is approximately one mMAG-LMA RTT (for the case of an MT handover from a fixed MAG to an mMAG or one MAG-LMA RTT, for the case of a handover from an mMAG to a fixed MAG) because updating the LMA with the new location of the MT is the only required signaling. We further consider a frequency of handovers ranging from one handover every 10 


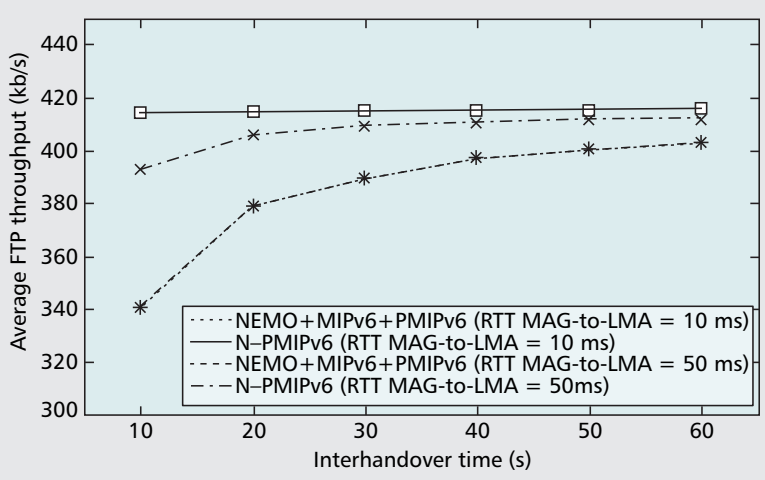

Figure 5. FTP throughput obtained by N-PMIPv6 compared with NEMO+MIPv6+PMIPv6.

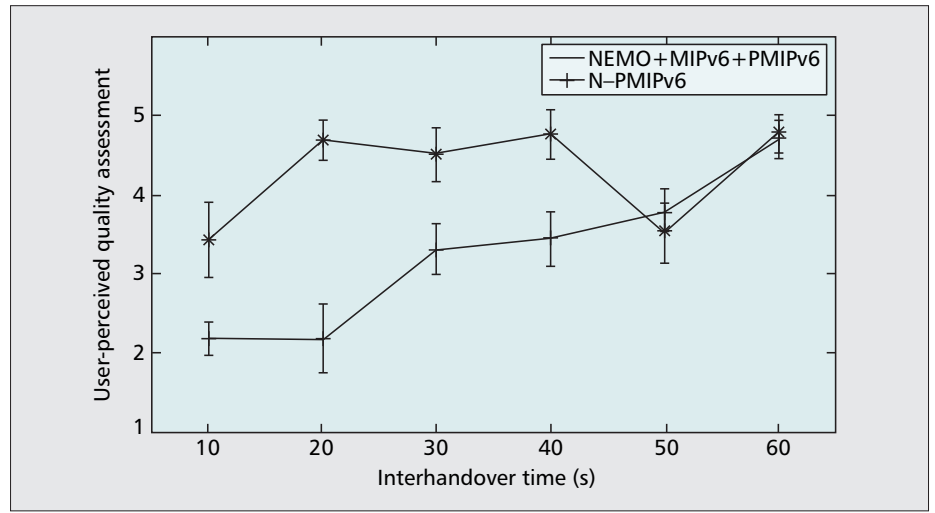

Figure 6. User-perceived video quality assessment.

\footnotetext{
${ }^{3}$ OPNET University Program;

http://www.opnet.com/services/university/

${ }^{4}$ http://www.videolan.org/

${ }^{5}$ http://www.netfilter.org/
}

seconds (highly dynamic scenarios) to one handover every 60 seconds (slowly changing scenarios).

We first analyzed the performance of a Transmission Control Protocol (TCP) data transfer by measuring the average throughput experienced when transferring a $20 \mathrm{MB}$ data file from the $\mathrm{CN}$ to the MT. Experiments were performed through simulations with the OPNET tool. 3 Two different values of RTT between the LMA and the MAGs (RTT MAG-to-LMA) were used in the simulations: $10 \mathrm{~ms}$ (usual case) and $50 \mathrm{~ms}$ (extreme case). This allowed us to evaluate the impact of the size of the N-PMIPv6 domain on the overall performance. The results obtained from the experiments with our approach and with NEMO+MIPv6+PMIPv6 are illustrated in Fig. 5. It can be observed that N-PMIPv6 improves the average throughput. Indeed, with NEMO+MIPv6+PMIPv6, each handover causes a severe interruption due to the latency associated with the signaling, thus degrading TCP performance. With N-PMIPv6, interruptions are much shorter because only local signaling is required and as a result, handovers do not degrade the throughput performance of TCP as much as in the case of NEMO+MIPv6+ PMIPv6.

The second application whose performance we analyzed is video streaming, in particular
VideoLAN Client (VLC), ${ }^{4}$ which transmits video over Real Time Protocol/User Datagram Protocol (RTP/UDP). The performance of this application was evaluated by means of real-life experiments with the following set up. Video was streamed from one PC to another, crossing a third PC. The iptables software ${ }^{5}$ was configured in the third PC to introduce interruptions of a duration and frequency equal to the ones caused by handovers (for the usual case). We conducted experiments with 16 real users who assessed the subjective video quality they perceived for each experiment. Following International Telecommunication Union (ITU) recommendations for the subjective evaluation of video and audio quality $[9,10]$, we asked the users to rate the quality of each video on a scale ranging from 5 (excellent quality) to 1 (bad quality). Figure 6 depicts the results obtained, in terms of average subjective quality and 95 percent confidence intervals.

The obtained results show that N-PMIPv6 clearly outperforms NEMO+MIPv6+PMIPv6, especially for highly dynamic environments (i.e., those in which an MT performs handovers very often). It can be seen that there is one point in the figure (one handover every 50 seconds) where the subjective quality with $\mathrm{N}$ PMIPv6 drops down to the level of NEMO+MIPv6+PMIPv6. The reason for this anomaly is that this particular experiment involved an unfortunate drop of some key packets that significantly degraded video quality despite the small number of lost packets. Nonetheless, results show that N-PMIPv6 performs significantly better due to the longer latency of NEMO+MIPv6+PMIPv6 handovers.

\section{CONCLUSIONS}

In this article we provide an overview of the major existing approaches to support mobile networks in network-based, localized mobility domains. Then, we propose N-PMIPv6, a novel architecture that extends these domains to include not only fixed points of attachment, but also mobile ones, achieving a better integration of mobile networks. N-PMIPv6, like PMIPv6, bases mobility support on network functionality, thus enabling conventional (i.e., not mobility-enabled) IP devices to change their point of attachment within an LMD without disrupting ongoing communications. As a result, N-PMIPv6 enables off-the-shelf IP devices to roam within the fixed infrastructure, attach to a mobile network and move with it, and also roam between fixed and mobile points of attachment while keeping the same IP address.

A key scenario for our architecture is the provision of Internet access from urban public transportation systems, such as undergrounds, suburban trains, and city buses. In these systems, providing connectivity from vehicles and stations is not the only requirement because this connectivity also must be maintained while changing vehicles.

Protocols already defined by the IETF could be combined to achieve a similar functionality to N-PMIPv6, although at the cost of introducing 
additional complexity at the user terminal. Furthermore, the experimental and simulation results provided in this article show that the performance of such a combination of protocols is substantially worse, from a user perspective, than with N-PMIPv6. Future plans include the implementation of N-PMIPv6 and the experimental evaluation of the state and processing overhead in the nodes of the architecture.

\section{ACKNOWLEDGMENT}

We would like to thank the users that kindly participated in the video experiments. We also thank the anonymous reviewers of this article for their valuable comments. The research leading to these results received funding from the European Community Seventh Framework Programme (FP7/2007-2013) under grant agreement no. 214994. This work also was partially supported by the Spanish government through the POSEIDON project (TSI2006-12507-C03).

\section{REFERENCES}

[1] H. Soliman et al. "Hierarchical Mobile IPv6 Mobility Management (HMIPv6)," RFC 4140, Aug. 2005.

[2] D. Johnson, C. Perkins, and J. Arkko, "Mobility Support in IPv6," RFC 3775, June 2004.

[3] J. Kempf, "Problem Statement for Network-Based Localized Mobility Management (NETLMM)," RFC 4830, Apr. 2007.

[4] S. Gundavelli et al., "Proxy Mobile IPv6," RFC 5213, Aug. 2008.

[5] V. Devarapalli et al., "Network Mobility (NEMO) Basic Support Protocol," RFC 3963, Jan. 2005.

[6] C. J. Bernardos et al., "VARON: Vehicular Ad-hoc Route Optimisation for NEMO," Comp. Commun., vol. 30, no. 8, June 2007, pp. 1765-84.

[7] C. Vogt and M. Zitterbart, "Efficient and Scalable, Endto-End Mobility Support for Reactive and Proactive Handoffs in IPv6," IEEE Commun. Mag., vol. 44, no. 6, June 2006, pp. 74-82.

[8] W. Matthews and L. Cottrell, "The PingER Project: Active Internet Performance Monitoring for the HENP Community," IEEE Commun. Mag., vol. 38, no. 5, May 2000, pp. 130-36.

[9] ITU-T Rec. P.800, "Methods for Subjective Determination of Transmission Quality," 1996.

[10] ITU-R Rec. BT.500-11, "Methodology for the Subjective Assessment of the Quality of Television Pictures," 2002.

\section{BIOGRAPHIES}

IGNACIO SOTo (isoto@it.uc3m.es) received a telecommunication engineering degree in 1993 and a Ph.D. in telecommunications in 2000, both from the University of Vigo, Spain. In 1999 he joined the University Carlos III of Madrid (UC3M), where he has been an associate professor since 2001. He was a research and teaching assistant in telematics engineering at the University of Valladolid from 1993 to 1999 . He has published several papers in technical books, magazines, and conferences, recently in the areas of mobility support in packet networks and heterogeneous wireless access networks.

CARLOS J. BERNARDOS (cjbc@it.uc3m.es) received a telecommu nication engineering degree in 2003 and a Ph.D. in telematics in 2006, both from UC3M, where currently he works as an associate professor. From 2003 to 2008 he worked at UC3M as a research and teaching assistant. His current work focuses on vehicular networks and IP-based mobile communication protocols. His Ph.D. thesis focused on route optimization for mobile networks in IPv6 heterogeneous environments. He served as TPC chair of WEEDEV 2009.

MARIA CALDERON (maria@it.uc3m.es) received a compute science engineering degree in 1991 and a Ph.D. degree in computer science in 1996, both from the Technical University of Madrid (UPM), Spain. She is an associate professor in the Telematics Engineering Department of UC3M. She has published over 25 papers in outstanding magazines and conferences in the fields of advanced communications, reliable multicast protocols, programmable networks, network mobility, and IPv6 mobility. Some of the recent European research projects in which she has participated are E-NEXT, LONG, GCAP, DAIDALOS, and GEONET.

AlBeRT BANCHS (banchs@it.uc3m.es) received his M.Sc. and Ph.D. degrees in telecommunications from the Technical University of Catalonia, Spain, in 1997 and 2002, respec tively. Since 2003 he has worked at UC3M. His research interests include performance evaluation and resource allocation of wireless networks. He worked for ICSI in 1997 for Telefonica I+D in 1998, and for NEC Network Laborato ries from 1998 to 2003. He is an Associate Editor for IEEE Communications Letters and has been a guest editor for IEEE Wireless Communications and Computer Networks.

ARTURo AzCorRa (azcorra@it.uc3m.es, arturo.azcorra @imdea.org) received his Ph.D. in 1989 from UPM. In 1992 he received an M.B.A. from Instituto de Empresa. He has been a full professor at UC3M since 1999. In 2006 he was appointed director of the international research institute IMDEA Networks. He has participated in many European research projects since the Third Framework Program and is currently the coordinator of the EU project CARMEN. He has published over 100 scientific papers in prestigious international magazines and conferences. 